\title{
EFECTO DE LA ANEMIA EN EL DESARROLLO INFANTIL: CONSECUENCIAS A LARGO PLAZO
}

\author{
Nelly Zavaleta ${ }^{1, a}$, Laura Astete-Robilliard ${ }^{1,2, b}$
}

\begin{abstract}
RESUMEN
La anemia en niños menores de tres años es un problema de salud pública en el Perú y el mundo. Se estima que la causa principal de la anemia, aunque no la única, es la deficiencia de hierro. Existen muchos estudios y revisiones sobre cómo esta carencia en los infantes impacta negativamente en el desarrollo psicomotor y, a pesar de corregirse la anemia, los niños con este antecedente presentan, a largo plazo, un menor desempeño en las áreas cognitiva, social y emocional. Estos hallazgos se describen en estudios observacionales, de seguimiento, así como en experimentales con grupo control. La anemia puede disminuir el desempeño escolar, y la productividad en la vida adulta, afectando la calidad de vida, y en general la economía de las personas afectadas. Se describen algunos posibles mecanismos de cómo la deficiencia de hierro, con o sin anemia, podría afectar el desarrollo en la infancia; por ello, causa preocupación la alta prevalencia de anemia que se observa en este grupo de edad. La prevención de la anemia en el primer año de vida debe ser la meta para evitar consecuencias en el desarrollo de la persona a largo plazo.
\end{abstract}

Palabras clave: Anemia; Desarrollo infantil; Efectos adversos a largo plazo (fuente: DeCS BIREME).

\section{EFFECT OF ANEMIA ON CHILD DEVELOPMENT: LONG-TERM CONSEQUENCES}

\begin{abstract}
Anemia in children younger than 3 years is a public health problem in Peru and worldwide. It is believed that one of the primary causes of anemia is iron deficiency. Numerous studies and reviews have reported that iron deficiency limited psychomotor development in children and that, despite the correction of anemia, children with iron deficiency experienced poorer long-term performance in cognitive, social, and emotional functioning. These outcomes were reported in observational studies, follow-up studies, and experimental studies with a control group. Anemia can decrease school performance, productivity in adult life, quality of life, and the general income of affected individuals. Here we describe possible mechanisms underlying the effect of iron deficiency, with or without anemia, on childhood development. The high rate of anemia in this age group is a cause for concern. Moreover, anemia should be prevented in the first year of life to avoid long-term negative effects on individual development.
\end{abstract}

Keywords: Anemia; Child development; Long term adverse effects (source: MeSH NLM).

\section{INTRODUCCIÓN}

La anemia es altamente prevalente en el mundo, afecta a casi la mitad de los niños menores de cinco años, y a una tercera parte de las gestantes ${ }^{(1)}$. En países de bajos y medianos ingresos, como el Perú, se estima que la causa principal de la anemia es la deficiencia de hierro ${ }^{(2)}$. La anemia es la etapa más severa de la deficiencia de hierro en el individuo ${ }^{(3)}$.
Los niños menores de dos años son particularmente vulnerables a la anemia, por su elevada velocidad de crecimiento y altos requerimientos de hierro, a lo que se suma las dietas bajas en hierro, mayores pérdidas de hierro por la presencia de parásitos, el bajo peso al nacer y episodios frecuentes de infecciones diarreicas ${ }^{(4)}$.

Algunas de las consecuencias inmediatas de la anemia son el retraso en el crecimiento, la respuesta inmunológica

\footnotetext{
Centro Nacional de Alimentación y Nutrición, Instituto Nacional de Salud. Lima, Perú.

Escuela de Nutrición, Universidad Peruana de Ciencias Aplicadas. Lima, Perú.

a Médico cirujano, master science en Nutrición; ${ }^{\mathrm{b}}$ nutricionista, magíster en Nutrición Pública

Recibido: 15/11/2017 Aprobado: 30/11/2017 En línea: 06/12/2017
} 
disminuida, regulación de la temperatura alterada ${ }^{(5)}$; algunos signos y síntomas como fatiga, debilidad y palidez (6); así como irritabilidad y déficit de atención ${ }^{(7)}$. La presencia de anemia en los menores de dos años tiene un efecto no solo en el desarrollo psicomotor, sino que sus consecuencias pueden manifestarse a lo largo del ciclo de vida.

Las consecuencias de la anemia no solo están relacionadas a la hipoxia, sino que pueden ser el producto de las alteraciones en la función del sistema nervioso central, que incluyen procesos como el metabolismo de los neurotransmisores, la sinapsis y la mielinización ${ }^{(7)}$. Es por eso que la anemia en la infancia podría afectar el desarrollo en el corto y largo plazo, limitando alcanzar el potencial de las personas afectadas.

\section{EPIDEMIOLOGÍA}

La anemia es el mayor problema de salud pública que afecta a la población en el mundo desarrollado y en desarrollo, no solo porque es el daño más común y el más ampliamente distribuido ${ }^{(1)}$, sino porque es el más prevalente entre los grupos vulnerables, como niños pequeños y mujeres en edad fértil ${ }^{(8)}$. Afecta al $43 \%$ de los menores de 5 años, $38 \%$ de las gestantes y al $29 \%$ de las no gestantes ${ }^{(1)}$.

En el Perú, en el año 2016, el 43,6\% de los niños de 6 a 35 meses $^{(9)}$, el $62,1 \%$ de los niños de 6 a 8 meses ${ }^{(2)}$ tuvieron anemia. La prevalencia de anemia en los menores de cinco años es $33,3 \%{ }^{(2)}$, y desde hace años se mantienen en valores similares, por ejemplo, en el 2009 la prevalencia de anemia en el mismo grupo de edad fue de $37,2 \%{ }^{(10)}$.

Por otro lado, en el 2016, tuvieron anemia el $20,8 \%$ de las mujeres entre 15 a 49 años residentes en nuestro país ${ }^{(2)}$, el $27,9 \%$ de las gestantes y el $23,5 \%$ de las mujeres que dan de lactar (2).

\section{ETIOLOGÍA DE LA ANEMIA}

La anemia tiene diversas causas (Figura 1), así, en zonas de no malaria la deficiencia de hierro podría significar hasta el $60 \%$ de la causa de anemia ${ }^{(11)}$. La inadecuada ingesta de hierro y otros nutrientes es una causa importante de anemia. Se han identificado otras causas de anemia, así en un estudio realizado en nuestro país en el año 2015, se determinó que las formas más frecuentes de anemia

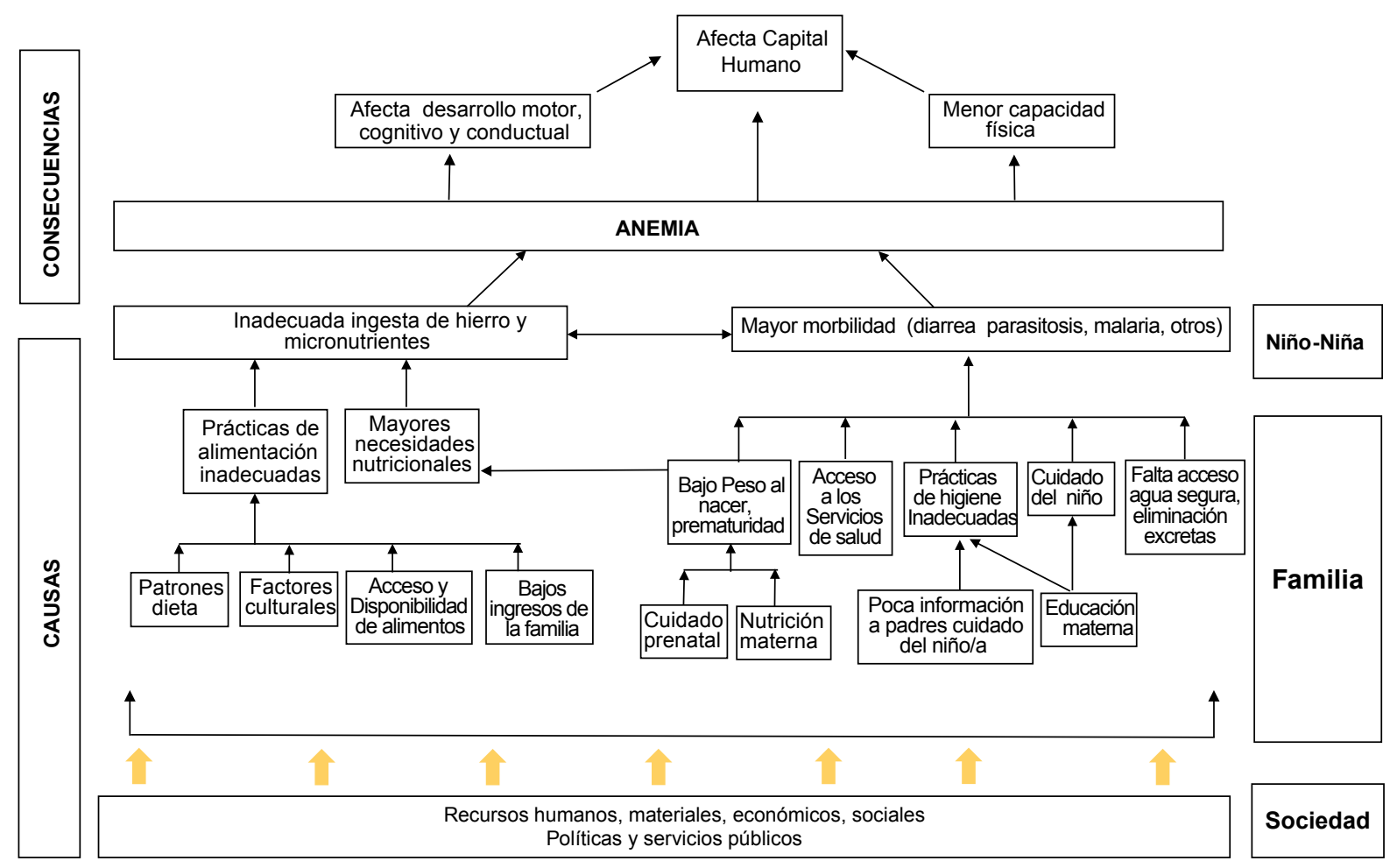

Fuente: elaboración propia

Figura 1. Causas y consecuencias de la anemia en población infantil 
son las relacionadas a parasitosis y aquellas que tienen al mismo tiempo dos o más causas ${ }^{(12)}$.

La anemia se asocia a diversos factores sociodemográficos, y factores relacionados al cuidado de la salud del niño. Un análisis secundario en el Perú (año 2016), encontró que en menores de 6 a 35 meses, la anemia está asociada tanto a factores sociodemográficos (lugar de residencia fuera de Lima, bajo nivel socioeconómico, madre adolescente y con poco nivel educativo, sexo masculino del menor, edad menor a 24 meses, fiebre reciente), como al cuidado madre-niño (falta de control prenatal y de tratamiento contra la anemia en la gestación, parto en el hogar, anemia materna durante la encuesta y ausencia de tratamiento antiparasitario en el menor) ${ }^{(13)}$ (Figura 1).

\section{EFECTOS DE LAANEMIA EN EL LARGO PLAZO}

\section{POSIBLES MECANISMOS DEL IMPACTO DE LAANEMIA EN EL DESARROLLO CEREBRAL}

Muchos de los estudios sobre la asociación del estado del hierro en la primera infancia y el desarrollo psicomotor y conductal, no hacen diferenciación entre anemia por deficiencia de hierro y deficiencia de hierro ${ }^{(14)}$. Se sabe que la carencia de hierro afecta negativamente el desarrollo cerebral, debido a que dicho proceso depende de enzimas y proteínas que contienen hierro ${ }^{(7)}$. Se conoce mucho de los mecanismos de los efectos a largo plazo de la anemia y la deficiencia de hierro, gracias a los resultados de los modelos realizados en animales, sobre todo en ratas.

Aunque sabemos que el hierro corporal en su mayoría, forma parte de dos proteínas que se encargan del transporte del oxígeno, la hemoglobina y la mioglobina, un pequeño porcentaje-aunque importante- de hierro es parte de otras enzimas y compuestos clave ${ }^{(15)}$. El hierro es un mineral contenido en enzimas que participan en la síntesis de lípidos que a su vez, son insumo para las membranas celulares y para la síntesis de mielina en el cerebro y por tanto, las funciones cognitivas y motoras se afectan negativamente ante un problema en la mielinización ${ }^{(14)}$.

El hierro también es parte de los procesos metabólicos de neurotransmisores principalmente dopaminérgicos y serotoninérgicos que tendrían un impacto en la conducta ${ }^{(7)}$. Todos estos procesos se llevan a cabo en el hipocampo, que es la región cerebral en la que se procesa el aprendizaje y la memoria ${ }^{(16)}$, así como comportamientos afectivos como la depresión y ansiedad ${ }^{(14)}$.

La anemia y la deficiencia de hierro, en especial, en periodos de rápido crecimiento como la etapa fetal y la infancia, afecta el desarrollo del hipocampo ${ }^{(16)}$ y la corteza frontal, alterando el sistema de neurotransmisión dopaminérgico. Es por ello que las etapas fetal y neonatal son consideradas cada vez más importantes en el desarrollo de la persona ${ }^{(7,14)}$.

\section{POSIBLE MECANISMO DE LA ANEMIA EN EL DESARROLLO INFANTIL}

El desarrollo infantil es un proceso de continuos cambios en el niño. En este análisis se centra la atención en solo tres de las áreas del desarrollo infantil, en las cuales se ha documentado ampliamente la asociación que existe con la anemia en las primeras etapas de vida; estas áreas son el desarrollo mental, motor y conductual del individuo ${ }^{(17,18)}$.

Desde hace varias décadas se conoce que la deficiencia de hierro impacta negativamente en el desempeño psicomotor y conductual ${ }^{(5,18)}$. Algunos estudios sugieren que estos efectos podrían tener un impacto a largo plazo, que no es posible revertir a pesar de haber superado la anemia, sin embargo, no hay consenso al respecto y se requieren más investigaciones.

\section{EFECTO DE LA ANEMIA EN EL DESARROLLO MOTOR}

El área motora se relaciona a la habilidad de los niños para controlar el movimiento de sus músculos, clasificándose en habilidades motoras finas y gruesas ${ }^{(19)}$. El desarrollo motor del niño es el resultado de muchos factores, propios del niño y otros de su entorno, siendo un riesgo muy importante la presencia de anemia durante el período crítico de los primeros años o meses de vida ${ }^{(18)}$.

En una revisión sistemática del 2001, se encontró que los niños que fueron anémicos durante los primeros años de vida, tuvieron luego un bajo desarrollo motor, caracterizado por deficientes habilidades motoras finas y gruesas. Sin embargo, sus efectos no necesariamente fueron reversibles luego del tratamiento de suplementación y a pesar que dicha deficiencia de hierro se había disipado. Con ese análisis se concluyó que, posiblemente, algunas variables ambientales expliquen parte o la totalidad de dichos resultados negativos, luego de la superación de la anemia ${ }^{(17)}$.

Un metaanálisis realizado por Sachdev et al. el 2005; en el que se controlan las variables ambientales, cuyo tratamiento con hierro incluyó suplementación oral y parenteral, así como la inclusión de alimentos fortificados, no encontró evidencia que sustente que luego del tratamiento, se mejore el desarrollo motor en los niños ${ }^{(20)}$.

Una revisión sistemática realizada por Szajewska et al. (21) el 2010, que buscó mejorar algunas de las limitaciones del metaanálisis previo, incluyó todos los estudios experimentales controlados y aleatorizados que compararon los efectos de la suplementación y la no suplementación, en madres gestantes no anémicas y niños saludables no anémicos, excluyendo a los sujetos con 
anemia. Se encontró que la suplementación de hierro en niños influye positivamente en su desarrollo psicomotor, pero no se encontró influencia en su desarrollo mental y su conducta $^{(21)}$ (Tabla 1). Cabe resaltar que el desarrollo motor es importante para posteriormente ejecutar funciones de lectura y escritura, que involucran tanto áreas cognitivas como motoras.

\section{EFECTOS DE LA ANEMIA EN EL DESARROLLO MENTAL}

El desarrollo mental o cognitivo incluye funciones de ejecución del pensamiento, memoria, razonamiento, atención, procesamiento visual, así como solución de problemas ${ }^{(19)}$. De manera similar que con el desarrollo motor, la anemia en la infancia reduce las habilidades cognitivas de los niños ${ }^{(18,20,21)}$. Sin embargo, una revisión sistemática del 2001, precisa que no está claro si el bajo desarrollo cognitivo y pobre desempeño escolar está asociado con la deficiencia de hierro en la infancia o se debe a un posible efecto de variables ambientales ${ }^{(18)}$. Por otro lado, en un metaanálisis del 2005 se reportó que luego de la suplementación con hierro, hubo una mejora leve del desarrollo mental ${ }^{(20)}$, siendo mayor entre quienes estaban inicialmente anémicos ${ }^{(20)}$. En otro metaanálisis, del 2010 , que cuenta con menos limitaciones metodológicas, se encontró que la suplementación de hierro en niños de 0 a 3 años podría no influenciar positivamente en su desarrollo mental ${ }^{(21)}$. En base a una revisión sistemática del 2014, se

Tabla 1. Algunas revisiones sistemáticas que evalúan el efecto de la suplementación de hierro sobre el desarrollo infantil

\begin{tabular}{|c|c|c|c|c|c|c|}
\hline Año & $\begin{array}{c}\text { Autor } \\
\text { (referencia) }\end{array}$ & Número de ECA & $\begin{array}{l}\text { Variables de } \\
\text { resultado }\end{array}$ & $\begin{array}{l}\text { Tratamiento / } \\
\text { prevención }\end{array}$ & $\begin{array}{l}\text { Número de } \\
\text { individuos }\end{array}$ & Conclusión \\
\hline 2011 & Hermoso M et al. ${ }^{(30)}$ & $\begin{array}{l}14 \text { ECA fueron } \\
\text { elegibles de } \\
\text { los cuales se } \\
\text { incluyeron } 7 \\
\text { estudios en niños } \\
\text { pequeños ( } 5 \text { de } \\
\text { ellos en menores } \\
\text { de } 6-24 \text { meses). }\end{array}$ & $\begin{array}{l}\text { Puntajes de } \\
\text { desarrollo } \\
\text { (desarrollo } \\
\text { psicomotor, } \\
\text { cognitivo y } \\
\text { desarrollo } \\
\text { mental) }\end{array}$ & $\begin{array}{l}\text { Alimentos } \\
\text { ricos en hierro, } \\
\text { alimentos } \\
\text { o fórmula } \\
\text { fortificados, } \\
\text { suplementación } \\
\text { con hierro } \\
\text { en niños y } \\
\text { adolescentes } \\
\text { hasta los } 18 \\
\text { años. }\end{array}$ & $\begin{array}{l}\text { Siete estudios } \\
\text { con } 1270 \\
\text { niños (5 ECA } \\
\text { corresponden a } \\
1008 \text { menores } \\
\text { de } 6 \text { a } 24 \\
\text { meses). }\end{array}$ & $\begin{array}{l}\text { Con la información disponible, } \\
\text { no se puede concluir } \\
\text { acerca del efecto de la } \\
\text { suplementación con hierro } \\
\text { en el desarrollo y la función } \\
\text { cognitiva en niños pequeños, } \\
\text { escolares y adolescentes, } \\
\text { aunque en } 3 \text { de las } 14 \text { ECA } \\
\text { evaluadas, hay alguna } \\
\text { evidencia que sugiere un } \\
\text { efecto positivo en el desarrollo } \\
\text { mental y psicomotor luego de } \\
\text { la suplementación de niños } \\
\text { anémicos (1-5 años de edad). }\end{array}$ \\
\hline 2010 & Szajewska H et al. (21) & $\begin{array}{l}2 \text { ECA en } \\
\text { gestantes y } \\
5 \text { ECA en } \\
\text { niños de 0-3 } \\
\text { años. Ambos } \\
\text { no anémicos } \\
\text { al inicio de la } \\
\text { suplementación }\end{array}$ & $\begin{array}{l}\text { Puntajes de } \\
\text { desarrollo } \\
\text { (desarrollo } \\
\text { psicomotor, } \\
\text { cognitivo y } \\
\text { desarrollo } \\
\text { mental, } \\
\text { coeficiente de } \\
\text { inteligencia y } \\
\text { desempeño } \\
\text { escolar). }\end{array}$ & $\begin{array}{l}\text { Suplementación } \\
\text { con hierro } \\
\text { elemental en } \\
\text { gestantes, } \\
\text { sulfato ferroso } \\
\text { o fórmula } \\
\text { fortificada en } \\
\text { niños. }\end{array}$ & $\begin{array}{c}430 \text { gestantes } \\
\text { ( } 216 \text { recibieron } \\
\text { hierro y } 214 \\
\text { placebo), y se } \\
\text { evaluaron a los } \\
\text { niños a los } 4 \\
\text { años (sólo } 302 \\
\text { niños). } \\
\text { Evaluaron } \\
\text { el desarrollo } \\
\text { mental en } 561 \\
\text { niños, y motor } \\
\text { en } \\
561 \text { niños. }\end{array}$ & $\begin{array}{c}\text { Con limitada evidencia, } \\
\text { podría sugerirse que la } \\
\text { suplementación con hierro } \\
\text { en la infancia (0-3 años) } \\
\text { influye positivamente en el } \\
\text { desarrollo psicomotor, pero } \\
\text { no en el desarrollo mental o la } \\
\text { conducta. No hubo efecto de } \\
\text { la suplementación prenatal en } \\
\text { el desarrollo mental o motor } \\
\text { de los niños. }\end{array}$ \\
\hline 2005 & Sachdev H et al. ${ }^{(20)}$ & $\begin{array}{c}\text { Se incluyeron } 17 \\
\text { ECA }\end{array}$ & $\begin{array}{l}\text { Puntajes de } \\
\text { desarrollo } \\
\text { (desarrollo } \\
\text { psicomotor, } \\
\text { cognitivo y } \\
\text { desarrollo } \\
\text { mental, } \\
\text { coeficiente de } \\
\text { inteligencia y } \\
\text { desempeño } \\
\text { escolar). }\end{array}$ & $\begin{array}{l}\text { Suplementación } \\
\text { con hierro oral } \\
\text { o parenteral, } \\
\text { fórmula y } \\
\text { cereales } \\
\text { fortificados. }\end{array}$ & $\begin{array}{l}\text { Se incluyeron a } \\
3646 \text { niños. } \\
\text { El desarrollo } \\
\text { mental se } \\
\text { evaluó en } 2827 \\
\text { niños ( } 1412 \\
\text { recibieron } \\
\text { hierro y } 1415 \\
\text { placebo), y } \\
\text { motor en } \\
1246 \text { niños (630 } \\
\text { recibieron hierro } \\
\text { y } 616 \text { placebo). }\end{array}$ & $\begin{array}{l}15 \text { estudios midieron el } \\
\text { desarrollo mental y } 10 \text { el } \\
\text { motor. La suplementación con } \\
\text { hierro mejora modestamente, } \\
\text { el puntaje de desarrollo } \\
\text { cerebral, en particular en } \\
\text { pruebas realizadas a niños de } \\
\text { más de } 7 \text { años. } \\
\text { El efecto de mejora del } \\
\text { desarrollo mental debido a la } \\
\text { suplementación con hierro no } \\
\text { se evidencia antes de los } 27 \\
\text { meses. }\end{array}$ \\
\hline
\end{tabular}

ECA: Ensayo clínico aleatorizado 
sabe que tanto la deficiencia de hierro, como la anemia con o sin deficiencia de hierro causan algún déficit cognitivo ${ }^{(22)}$.

En una cohorte de Costa Rica se observó que los individuos con anemia en la infancia no alcanzan el mismo nivel de desarrollo cognitivo que aquellos con un adecuado nivel del hierro, y aquellos que pertenecían a niveles socioeconómicos más bajos presentaron una brecha de desempeño cognitivo de 10 puntos menos que en la niñez, esta se hizo mayor a los 19 años con una brecha de 25 puntos ${ }^{(23)}$. Esta cohorte evaluó a 185 niños enrolados entre los 12 y 23 meses de edad, cuyos datos se han analizado a los 5, 11 a 14,15 a 18 y 19 años de edad ${ }^{(23)}$.

Por otro lado, en una cohorte realizada en Chile, se encontró que en adultos que tuvieron anemia en su primer año de vida y que luego fue corregida mediante suplementación, se presentaron patrones alterados de la conectividad cerebral a la edad promedio de 21,5 años, lo que sugiere que la anemia produce algún efecto en el desarrollo mental a largo plazo ${ }^{(24)}$, a pesar de la corrección de los niveles de hemoglobina.

\section{EFECTOS DE LA ANEMIA EN LA CONDUCTA}

La conducta socioemocional es otra de las áreas del desarrollo infantil, y comprende el establecimiento de relaciones con otras personas usando sus emociones, así como la regulación de sí mismo y su interés hacia el exterior ${ }^{(19)}$. En niños que tuvieron anemia en el primer año de vida (a los 6, 12 y 18 meses), y que luego fue corregida mediante suplementación, se encontró que a los 10 años de seguimiento, tuvieron tiempos de reacción más lentos y menor capacidad para controlar respuestas impulsivas, lo que se le denomina como pobre control inhibitorio (25). Esta dificultad es uno de los déficits conductuales que se relaciona con trastornos como déficit de atención e hiperactividad, entre otros ${ }^{(26)}$. Estos comportamientos no deseados y problemas de conducta tienen un impacto no solo dentro de cada hogar, sino también en las escuelas, y posiblemente, con el tiempo, generen algún tipo de problema social mayor.

Por otro lado, un estudio en China encontró que los niveles bajos de hemoglobina en niños de cuatro años, estuvieron asociados a problemas de conducta externalizada a los 6 años, asociación que no se vio afectada con problemas de adversidad social ${ }^{(27)}$. Hubo, además, una diferencia según sexo en la manifestación conductual a los seis años, los niños tenían más problemas de atención, mientras que las niñas presentaron mayor agresión (27). Este estudio presenta algunas limitaciones como el poco tamaño de muestra, se reporta solo niveles bajos de hemoglobina y no anemia, y no mencionan otras medidas que evalúan la malnutrición como el peso y talla ${ }^{(27)}$. Entre las limitaciones de las revisiones sistemáticas podemos mencionar que combinaban muchos tipos de evaluaciones (mental y motora), otros no consideraron la diferencias de edad, ni los instrumentos con los cuales se evaluó el desarrollo mental o motor ${ }^{(20)}$. Cabe señalar que se asumió en todos los casos, que la anemia fue por deficiencia de hierro cuando no necesariamente es así. Finalmente, no se incluyeron trabajos no publicados (sesgo de publicación), lo que puede significar que justamente esos trabajos no publicados son los que reportan resultados "no favorables" a la hipótesis que puede ser la deseada.

\section{EFECTOS DE LA ANEMIA A LARGO PLAZO EN SALUD, EDUCACIÓN Y OTROS}

La anemia genera al Estado Peruano un costo alto. En un estudio en Perú se describió que la anemia genera una pérdida del $0,62 \%$ del producto bruto interno (PBI), lo que para el año 2009-2010 significó un aproximado de USD 857 millones (valor estimado a partir del tipo de cambio del dólar del día 1 de septiembre de 2017 [ 1 USD = 3,239 soles]), cifra que representó cerca del $40 \%$ del presupuesto del sector Salud de ese mismo año ${ }^{(28)}$. Casi la mitad del costo que genera la anemia al Estado peruano $(46,3 \%)$ es por pérdida cognitiva, un 12,7 y $18,2 \%$ por pérdidas de escolaridad y productividad en el adulto, respectivamente ${ }^{(28)}$.

Esto afecta, principalmente, a los sectores de Educación, Empleo, Producción, Agricultura y Minería, ya que la anemia resta capacidades en el trabajo, ocasionando pérdidas en la productividad, lo que se traduce en un menor acceso a puestos de trabajo, o en un menor salario. Todo ello va a influir en la cantidad de dinero que el Estado recauda a través del pago de impuestos.

Alcázar L. describe que el $13 \%$ del costo de éstas pérdidas por anemia corresponden a la atención de partos prematuros, $9 \%$ debido al retraso escolar, y 0,6 y $0,2 \%$ por el tratamiento de la anemia de menores de 6 a 36 meses y en gestantes, respectivamente ${ }^{(28)}$. Esto puede generar costos elevados en el sector Salud, lo que resta la posibilidad de brindar servicios de calidad, o que no se mejore la cobertura en las prestaciones de salud.

\section{CONCLUSIONES}

Considerando que cada año se reportan aproximadamente 600 mil nacimientos en el Perú ${ }^{(29)}$, se estima que 404 938 niños tienen anemia en el primer año de vida, los cuales estarían en riesgo de no alcanzar su potencial de desarrollo infantil a causa de esta enfermedad. Se hace necesario, fortalecer las acciones para alcanzar una mayor adherencia y efectividad de la suplementación con hierro a la madre y al niño, promover la alimentación adecuada tanto en el niño como en la gestante y mejorar la atención sanitaria para reducir la carga de enfermedad infantil y así contribuir a prevenir la anemia en el primer año de vida. 
Contribuciones de autoría: NZ participo en el diseño del estudio, revisión de bibliografía, desarrollo y revisión del manuscrito. LAR participo en la revisión de bibliografía, desarrollo y revisión del manuscrito. Ambas aprobamos la versión final.
Fuentes de financiamiento: la investigación ha sido financiada por el Instituto Nacional de Salud de Perú.

Conflictos de interés: los autores declaran no tener conflicto de interés.

\section{REFERENCIAS BIBLIOGRÁFICAS}

1. World Health Organization (WHO). The global prevalence of anaemia in 2011 [Internet]. Ginebra: World Health Organization; 2015. 48 p. [citado septiembre 2017]. Disponible en: www. who.int/about/licensing/copyright form/en/index.html

2. Instituto Nacional de Estadística e Informática. Encuesta Demográfica y de Salud Familiar 2016 Nacional y Regional (ENDES 2016) [Internet]. Lima: Instituto Nacional de Estadística e Informática; 2017. Disponible en: http://proyectos. inei.gob.pe/endes/resultados.asp

3. United Nations Children's Fund, United Nations University, World Health Organization. Iron deficiency anaemia. Assessment, prevention and control. A guide for programme managers [Internet]. Geneva: World Health Organization; 2001. Disponible en: http://www.who. int/nutrition/publications/en/ida_ assessment_prevention_control.pdf

4. World Health Organization. Guideline: Intermittent iron supplementation in preschool and school-age children. Geneva: WHO; 2011. Disponible en: https://www.ncbi.nlm.nih.gov/books/ NBK179850/

5. Thomas DG, Grant SL, AubuchonEndsley NL. The Role of Iron in Neurocognitive Development. Dev Neuropsychol. 2009;34(2):196-222. doi: 10.1080/87565640802646767.

6. MacLean E, Egli I, Benoist B, Wojdyla D, Cogswell M. Worldwide prevalence of anemia in pre-school age children, pregnant women and non-pregnant women of reproductive age. En: Kraemer K, Zimmermann M, editors. Nutritional Anemia. Basel: Sight and life Press; 2007.

7. Vallée L. Fer et neurodéveloppement. Arch Pediatr. 2017;24(5S):5S18-5S22. doi: 10.1016/S0929-693X(17)24005-6.

8. Miller JL. Iron deficiency anemia: a common and curable disease. Cold Spring Harb Perspect Med. 2013;3(7). pii: a011866. doi: 10.1101/cshperspect. a011866.

9. Instituto Nacional de Estadística e Informática (INEI). Indicadores de Resultados de los Programas Presupuestales, Primer Semestre 2017 Encuesta Demográfica y de Salud Familiar (Resultados Preliminares al 50\% de la muestra) [Internet]. Lima: INEI; 2017. Disponible en: http://proyectos.inei.gob.pe/endes/ppr.asp

10. Instituto Nacional de Estadística e Informática (INEI). Encuesta Demográfica y de Salud Familiar ENDES 2009 [Internet]. Lima: INEI; 2010. Disponible en: http://encuestas. inei.gob.pe/endes/

11. Rastogi T, Mathers C. Global burden of Iron Deficiency Anaemia in the year 2000 [Internet]. 2002 [cited 2017 Aug 30]. Available from: http://www. who.int/healthinfo/statistics/bod_ irondeficiencyanaemia.pdf

12. Gonzales E, Huamán-Espino L, Gutiérrez C, Aparco JP, Pillaca J. Caracterización de la anemia en niños menores de cinco años de zonas urbanas de Huancavelica y Ucayali en el Perú. Rev Peru Med Exp Salud Publica. 2015;32(3):431-9.

13. Velásquez-Hurtado JE, Rodríguez Y, Gonzáles M, Astete-Robilliard L, LoyolaRomaní J, Vigo WE, et al. Factores asociados con la anemia en niños menores de tres años en Perú: análisis de la Encuesta Demográfica y de Salud Familiar, 20072013. Biomedica. 2016;36(2):220-9. doi: 10.7705/biomedica.v36i2.2896.

14. Murray-Kolb LE. Iron and brain functions. Curr Opin Clin Nutr Metab Care. 2013;16(6):703-7. doi: 10.1097/ MCO.0b013e3283653ef8.

15. Bowman BA, Russell RM, editors. Hierro. Conocimientos actuales sobre nutrición. 8va edició. Washington DC: Instituto Internacional de Ciencias de la Vida; 2003. p. 873.

16. Muñoz P, Humeres A. Iron deficiency on neuronal function. Biometals. 2012;25(4):825-35. doi: 10.1007/ s10534-012-9550-x.

17. Walter T. Effect of Iron-Deficiency Anemia on Cognitive Skills and Neuromaturation in Infancy and Childhood. Food Nutr Bull. 2003;24(4 Suppl):S104-10
18. Grantham-McGregor S, Ani C. A review of studies on the effect of iron deficiency on cognitive development in children. J Nutr. 2001;131(2S-2):649S-666S; discussion 666S-668S

19. Frongillo EA, Tofail F, Hamadani JD, Warren AM, Mehrin SF. Measures and indicators for assessing impact of interventions integrating nutrition, health, and early childhood development. Ann N Y Acad Sci. 2014;1308:68-88. doi: $10.1111 /$ nyas. 12319 .

20. Sachdev H, Gera T, Nestel P. Effect of iron supplementation on mental and motor development in children: systematic review of randomised controlled trials. Public Health Nutr. 2005;8(2):117-32.

21. Szajewska H, Ruszczynski M, Chmielewska A. Effects of iron supplementation in nonanemic pregnant women, infants, and young children on the mental performance and psychomotor development of children: a systematic review of randomized controlled trials. Am J Clin Nutr. 2010;91(6):1684-90. doi: 10.3945/ ajcn.2010.29191.

22. Jáuregui-Lobera I. Iron deficiency and cognitive functions. Neuropsychiatr Dis Treat. 2014;10:2087-95. doi: 10.2147/ NDT.S72491.

23. Lozoff B, Jimenez E, Smith JB. Double burden of iron deficiency in infancy and low socioeconomic status: a longitudinal analysis of cognitive test scores to age 19 years. Arch Pediatr Adolesc Med. 2006;160(11):1108-13.

24. Algarin C, Karunakaran KD, Reyes S, Morales C, Lozoff B, Peirano P, et al. Differences on Brain Connectivity in Adulthood Are Present in Subjects with Iron Deficiency Anemia in Infancy. Front Aging Neurosci. 2017;9:54. doi: 10.3389/fnagi.2017.00054.

25. Algarín C, Nelson CA, Peirano P, Westerlund A, Reyes S, Lozoff B. Iron-deficiency anemia in infancy and poorer cognitive inhibitory control at age 10 years. Dev Med Child Neurol. 2013;55(5):453-8. doi: $10.1111 / \mathrm{dmcn} .12118$. 
26. Mediavilla-García C. Neurobiología del Trastorno de Hiperactividad. Rev Neurol. 2003;36(6):555-65.

27. Su J, Cui N, Zhou G, Ai Y, Sun G, Zhao SR, et al. Hemoglobin Status and Externalizing Behavioral Problems in Children. Int J Environ Res Public Health. 2016;13(8). pii: E758. doi: 10.3390/ ijerph13080758.

28. Alcázar L. Impacto Económico de la anemia en el Perú [Internet]. Lima: GRADE, Acción contra el Hambre; 2012. Disponi- ble en: http://www.grade.org.pe/upload/ publicaciones/archivo/download/pubs/ LIBROGRADE_ANEMIA.pdf

29. Instituto Nacional de Estadística e Informática. Perú: Natalidad, mortalidad y nupcialidad, 2015 [Internet]. Lima: Instituto Nacional de Estadística e Informática; 2016. Disponible en: http://www.inei. gob.pe/media/MenuRecursivo/publicaciones_digitales/Est/Lib1407/libro.pdf

30. Hermoso M, Vucic V, Vollhardt C, Arsic A, Roman-Viñas B, Iglesia-Altaba I, et al.
The effect of iron on cognitive development and function in infants, children and adolescents: a systematic review. A Ann Nutr Metab. 2011;59(2-4):154-65. doi: $10.1159 / 000334490$.

Correspondencia: Nelly Zavaleta Dirección: Jr. Tizón y Bueno 276, Jesús Maria, Lima 11, Perú

Teléfono: (+51) 975451637

Correo electrónico:nzavaleta@ins.gob.pe

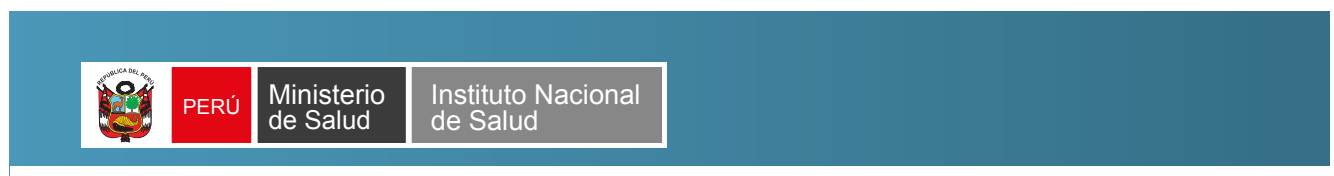

\section{Inclusión social en salud: acercando el diagnóstico de dengue a las poblaciones afectadas}

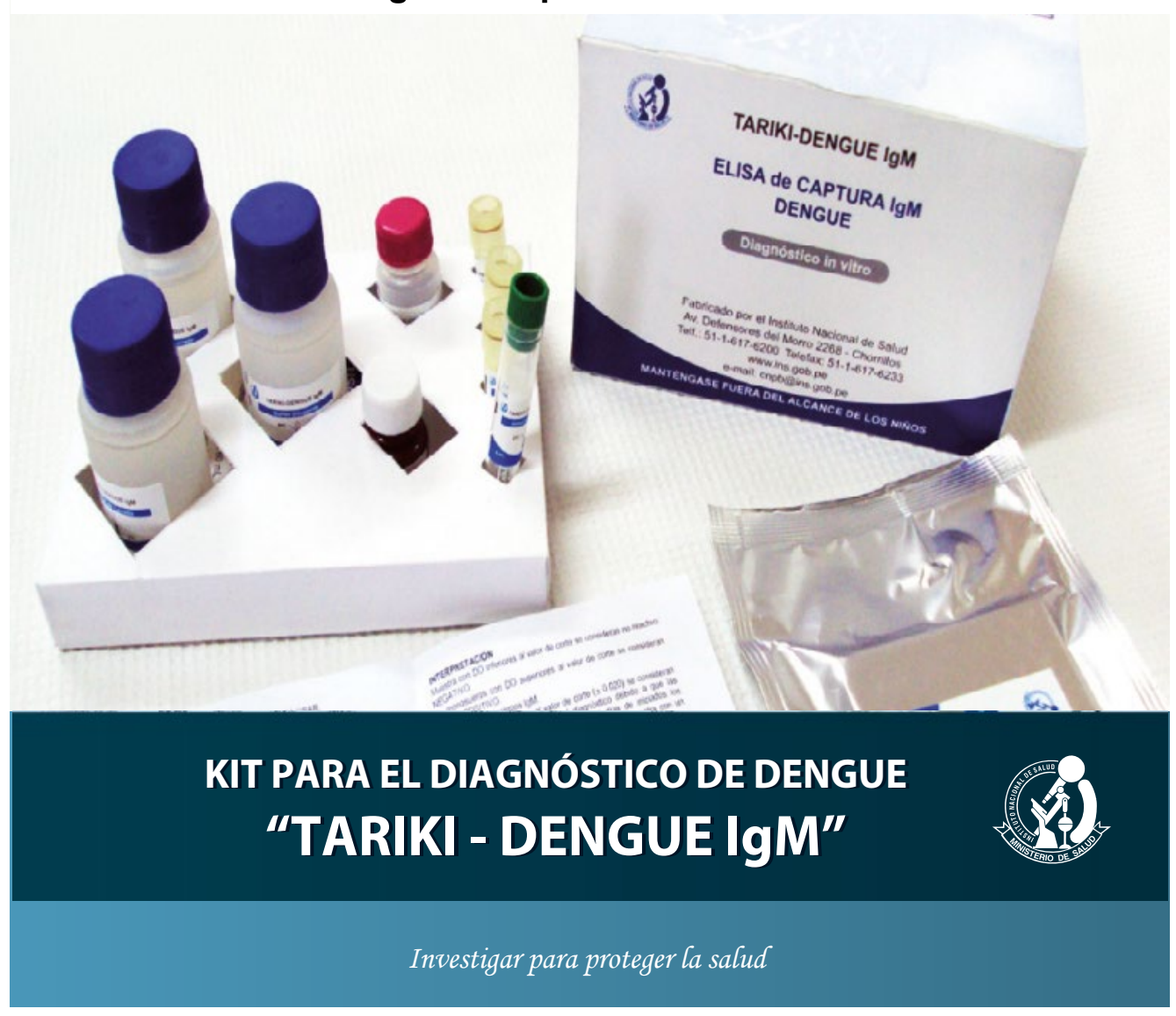

